\title{
Supernumerary Marker chromosome and Global Developmental Delay: Role of Microarray
}

\author{
SUMITRA BACHANI, VATSLA DADHWAL, DEEPIKA DEKA
}

Key Message: Marker chromosome may result in a structural or functional abnormality, however $77 \%$ can be associated with a normal phenotype. Array CGH must be used to establish the nature of supernumerary marker chromosome. If associated with global developmental delay the cause may lie in a chromosome other than the marker.

\section{Introduction:}

Small supernumerary marker chromosomes (sSMCs) are defined as structurally abnormal chromosomes that cannot be identified or characterized by conventional karyotype analysis and are generally equal in size or smaller than chromosome number 201. sSMCs are present in nearly $0.044 \%$ of live births and $0.075 \%$ of prenatal cases 2,3 . Approximately $77 \%$ of sSMCs arise de novo and $23 \%$ are inherited 2,3 . Nearly $70 \%$ of sSMCs are derived from the short arms and pericentromeric regions of acrocentric chromosomes. 4 Most (70\%) of de novo sSMCs have no phenotypic effects5. However, in at least 30-50\% of prenatally detected SSMC cases, the pregnancy is terminated ${ }^{2,3}$ which means unnecessary abortions were induced in a certain percentage of potentially healthy children with SSMC.

Therefore the precise characterization of marker chromosomes is crucial for prenatal diagnosis and proper genetic counselling. Here we present a case and the molecular characterization of an SSMC derived from chromosome 15 in prenatal diagnosis.

Case: Mrs X ,38 yrs female, G3P1A1L1 presented at 17 weeks of gestation with history of her girl child having Global development delay. Her period of gestation was corresponding to the Ultrasonography . She had undergone combined first trimester screening by which was reported to be low risk. There was no gross congenital malformation of the fetus in present pregnancy.

She had delivered a girl child six years back at term by an Emergency Cesarean section for non progress of labour. The child had delayed milestones and mild facial dysmorphism for which she was evaluated and diagnosed to have Global developmental delay. Genetic evaluation was done including $\mathrm{CGH}$ array and FISH which diagnosed the presence of a marker chromosome in the child, 47,XX + mar. On parental evaluation the paternal karyotype was normal $46 \mathrm{XY}$ and mother was detected to have the same marker chromosome. However the mother was phenotypically and functionally normal and part of the medical profession. A $2.05 \mathrm{Mb}$ duplication was detected on the long arm of chromosome 15 (cytogenetic location 15q11.1q11.2) of mother.

Since both the mother and the child had the identical marker chromosome and mother being normal , the Global development delay could not be completely attributed to the marker chromosome. The microarray report of both proband and the mother was awaited. Meanwhile the parents opted for prenatal testing by Amniocentesis and full Karyotype and FISH studies in the fetus of present pregnancy. She underwent an uneventful procedure and was discharged the same day.FISH was performed on the cells from amniotic fluid sample using Vlysis DNA probe LSI SNRPN/

1. Dr Sumitra Bachani MD FICOG FICMCH Fellow MFM(AIIMS Delhi) Specialist \& Assistant Professor OBGYN, Vardhman Mahavir Medical College\&Safdarjung Hospital, New Delhi

2. Dr Vatsla Dadhwal MD FICOG FIMSA, Professor, Division of Maternal Fetal Medicine ,OBGYN Department of Obstetrics \& Gynecology, All India Institute of Medical Sciences, New Delhi

3. Dr Deepika Deka MD, FICOG, FIMSA, FICMCH, Professor \& Unit Head, Fetal Medicine Division, Department of Obstetrics \& Gynecology, All India Institute of Medical Sciences, New Delhi

Address of Correspondence: Dr Sumitra Bachani, Flat no 1485, Sector B Pocket 1.Vasant Kunj New Delhi, India 100070, Phone number: +919212129161, E-mail: sumitrabachani@gmail.com 
LSIPML/CEP (tricolor). Signal enumeration was done in 50 cells.(Image 1) The final

report of the amniotic fluid karyotype was the same as the mother and the index case.(Fig. 1)

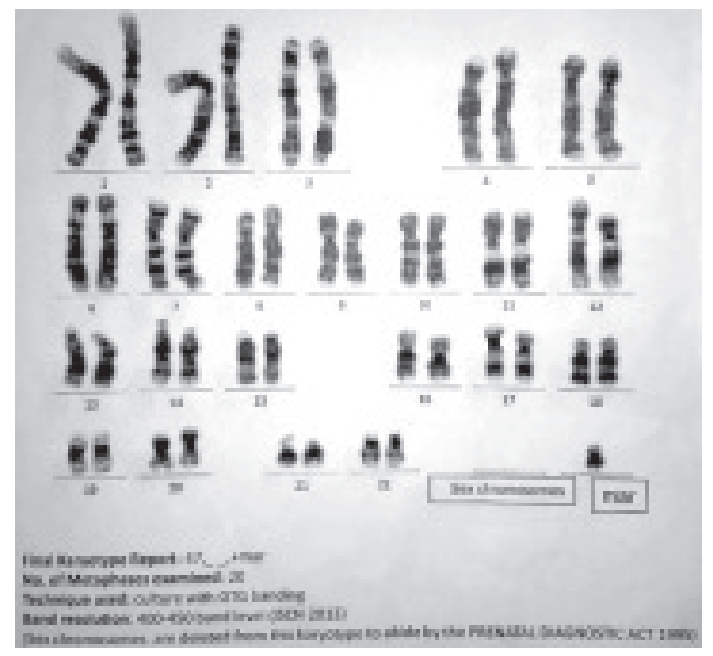

Fig.-1: Karyotype (amniotic fluid)

Despite counseling the couple refused further analysis and opted for termination. The abortus underwent an autopsy wherein no structural abnormality was detected.

The final microarray reports of the proband,fetus and mother was reported as follows.

Fetus: Genomic duplication of $2.6 \mathrm{Mb}$ on cytoband $15 q 11.1 q 11.2$ starting from

$20.055,137 \mathrm{bp}$ to $22,698,581 \mathrm{bp}$ on long arm of Chromosome \#15. This has been reported benign on ISCA Database.(Fig. 2)

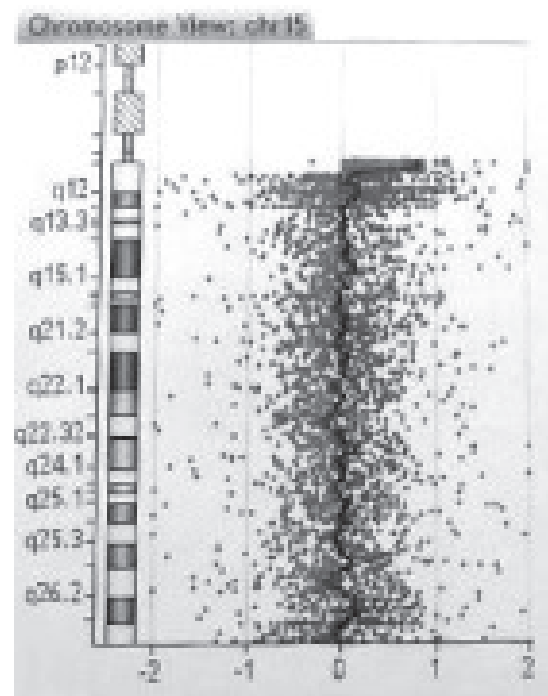

Fig.-2: Microarray Fetus
Mother: Genomic duplication of $2.6 \mathrm{Mb}$ on cytoband $15 q 11.1 q 11.2$ starting from

$20,095,481$ to $22,698,581$ bp on long arm $q$ of chromosome\#15. This has been reported benign on ISCA Database.(Image 3)

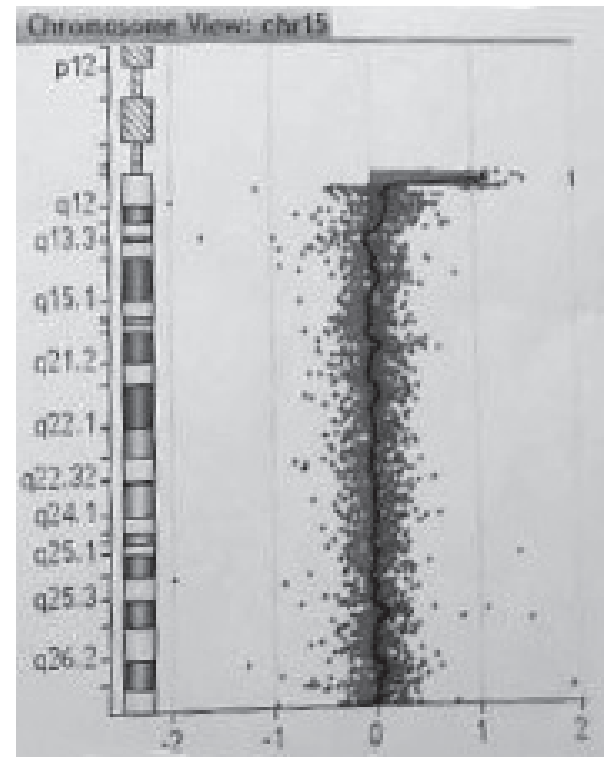

Fig.-3: Microarray mother

$\mathrm{LOH}$ (Loss of heterozygosity) of $19.2 \mathrm{Mb}$ on Chromosome \#16. It may be due to parental consanguinity which was absent in this case and can lead to uniparental disomy.

Proband: Genomic duplication of $2.6 \mathrm{Mb}$ on cytoband $15 q 11.1 q 11.2$ starting from $20.055,137$ bp to $22,698,581 \mathrm{bp}$ on long arm of Chromosome\#15. This has been reported benign by ISCA Database.

Genomic duplication of $177 \mathrm{~Kb}$ on cytoband 9p 24.3 starting from 204,193 bp to 381,489 on short arm $p$ of chromosome \# 9. Reported uncertain likely benign by ISCA. (Fig. 4)

Review of Literature: It is estimated that $50 \%$ of all SMC are derived from chromosome $15^{6}$. Children with an SSMC are individually very different from each other, even when the sSMC causes a known syndrome. Wong et al reported a de novo marker chromosome part of 15 detected on amniocentesis in a 21 year old woman with high risk for Trisomy 21 on combined screen. Parental Karyotype was normal and the fetus did not have any anomaly or dysmorphism on sonography. Pregnancy outcome was successful with the baby having karyotype 


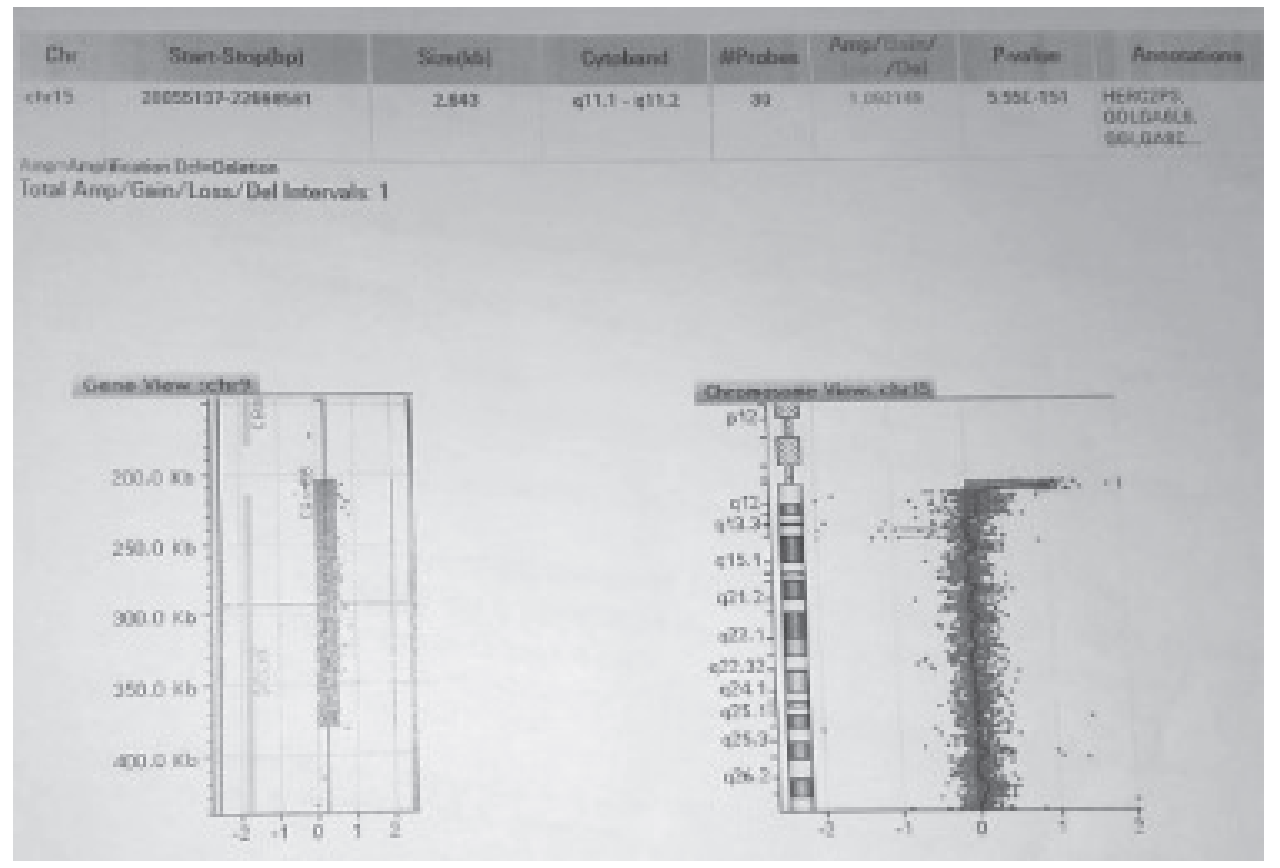

Fig.-4: Microarray of proband

$47 \mathrm{XY}+$ Marker.At one year of age, the baby was progressing normally.7 Jon Soo Kim et al reported a 10-month-old female infant referred to their pediatric neurology clinic for uncontrolled seizures and global developmental delay. Array CGH showed an amplification from $15 q 11.1$ to $15 q 13.1$ spanning 8.47 Mb. 6 The reported clinical features of patients with $15 q 13.3$ microdeletion and duplication are rather heterogeneous, ranging from mental retardation and psychiatric illness to seizures.

In the present case the proband and the mother had the same marker chromosome on routine karyotype and FISH however the mother was phenotypically and functionally normal.

Approximately $70 \%$ of SMCs are de novo and $30 \%$ are inherited. The most common SMCs are derived from acrocentric chromosomes and have a satellited or bisatellited structure. Chromosome 15 accounts for the highest percentage ( $50 \%)$ of this group. Melo et al reported a case of a 39-year-old woman who underwent amniocentesis at 16 weeks of gestation for advanced maternal age. An abnormal fetal karyotype -

$47, \mathrm{XX}$,+mar - with one sSMC was detected in all metaphases. Cytogenetic and molecular analyses revealed a fetal karyotype $47, \mathrm{XX},+$ mar pat.ish idic(15)(ql2)(D15Zl+ +,SNRPN"), in which the sSMC(15) was a paternally inherited inverted duplicated chromosome and did not contain the critical region of Prader-Willi/Angelman syndromes. Uniparental disomy was excluded and genetic sonogram was normal, pregnancy outcome was a normal baby. This case supports the literature in two aspects: sSMC(15)s that do not contain PWACR generally have a normal phenotype, and sSMCs transmitted by normal carriers to their offspring are not commonly correlated with clinical problems.8Some sSMCs lead to specific syndromes. Entire and partial gene deletions/ duplications can produce a completely different phenotypic effect. The low copy repeats (LCRs) in chromosome 15q11q13 have been recognized as Breakpoints (BP) for not only intrachromosomal deletions and duplications but also small supernumerary marker chromosomes 15 , sSMC (15). 9 Most sSMC (15) s take the form of a dicentric inv dup and can be classified into two groups: small sSMC (15) s and large sSMC (15) s. The small sSMC (15) s are metacentric chromosomes without euchromatic material and do not contain the Prader-Willi/Angelman Critic Region (PWACR), which usually clinically irrelevant. In contrast, the large sSMC (15) s are acrocentric chromosomes containing copies of PWACR and are frequently associated with abnormal phenotype.( mELO ) For the other sSMCs, it is still not possible to predict outcomes, although new cases can now 
be compared with the cases collected on the SSMC website. 9,10

If a child has problems with growth or development, it is likely that the sSMC has affected them but the cause could also be something different, not the sSMC. The correct characterization of gene deletions and duplications is a crucial point in order to identify the genotype phenotype correlation. Some conclusions can be drawn by comparing other cases from the medical literature.Conventional karyotype analysis can detect numerical and structural chromosomal abnormalities but cannot determine the origin and genetic content of sSMCs. Array CGH has the ability to detect DNA dosage imbalance including deletions and duplications in the euchromatic regions and is useful for the characterization of the origin and hereditary effects in the SSMC. ${ }^{11}$

Sun et al reported the analysis of twenty cases with sSMC initially were detected by G-binding karyotype. Routine cytogenetic analysis showed mosaic marker chromosome in six out of the 20 cases. All the 20 cases were subjected to aCGH assay, and seventeen of them were successfully identified for the chromosome origin. Array $\mathrm{CGH}$ has many advantages that make it extremely useful for characterizing SSMCs. It clearly and easily determines the components of sSMCs in a single assay. This advantage was especially useful for complex sSMCs and multiple sSMCs. Complex sSMCs is a subgroup of sSMCs which consist of chromosomal materials derived from more than one chromosome.12 Vetro et al reported 4 cases of sSMC,s that after array $\mathrm{CGH}$ were interpreted rather differently reporting two types of complex markers which DNA content was overlooked by conventional approaches. All are likely derived from partial trisomy rescue events thus changing the present ideas on composition of supernumerrary marker chromosome. ${ }^{13}$

In the present case the proband also had an additional Genomic duplication in the short arm of chromosome \#9. Glessner et al in the first metaanalysis of copy number variations(CNVs) studied five cohorts including Autism spectrum disorders(ASD) and Attention deficit hyperactivity disorders(ADHD). They stated that cumulative evidence indicates a shared genetic etiology of neurodevelopmental and neuropsychiatric diseases.
They identified the DOCK8/KANK1 locus as containing exonic $\mathrm{CNV}$ s with genome-wide significant meta $P$ values and consistent direction of effects across all five cohorts. There was a significant association of genomic duplication in

Chromosome \#9 short arm with ADS, ADHD and Neuropsychiatric illnesses. ${ }^{14}$

In the present case though the parents opted for termination based on the FISH report

,the array CGH would have given the clarity towards whether the fetus would have been actually affected as its microarray did not show the genomic duplication in chromosome \#9 present in the proband. The marker chromosome was only co incidental to the developmental delay.

Presence of uniparental disomy as a cause for ssSMC, $s$ is being investigated. In a reveiw article Kotzot stated that the incidence of UPD in cases with a SMC is increased by coincidence, ascertainment bias, and because for most chromosomes only then is the fetus viable. 15 In this case study we validate that array $\mathrm{CGH}$ analysis provides an alternative to telomere FISH and disease-specific FISH in the cytogenetic diagnostic laboratory.

Conclusion We conclude that array $\mathrm{CGH}$ is a modern and precise diagnostic tool that will complement and enhance current methods of detecting chromosomal imbalances prenatally. In combination of a detailed ultrasound examination and karyotype analysis, it can provide more precise and rapid prenatal diagnosis of SSMC. It is suggested that UPD is always tested for when an SSMC is detected.In order to establish a stronger base for clinical service in the future and avoid miss characterisation, more sSMC cases need to be characterised in detail. This will help to clarify the variable clinical characteristics of SSMCs and provide additional information to aid clinical service and future research.

\section{References:}

1. Liehr T. Characterization of prenatally assessed de novo small supernumerary marker chromosomes by molecular cytogenetics. Methods Mol Biol 2008.; 444: 27-38.

2. Liehr T. Handling small supernumerary marker chromosomes in prenatal diagnostics. Expert Rev Mol Diagn 2009; 9: 317-324. 
3. Liehr T, Weise A. Frequency of small supernumerary marker chromosomes in prenatal, newborn, developmentally retarded and infertility diagnostics. Int J Mol Med 2007; 19: 719-731.

4. Viersbach R, Engels $H$, Gamerdinger $U$, Hansmann M. Delineation of supernumerary marker chromosomes in 38 patients. Am J Med Genet 1998; 76: 351-358.

5. Warburton D. De novo balanced chromosome rearrangements and extra marker chromosomes identified at prenatal diagnosis: clinical significance and distribution of breakpoints. Am J Hum Genet 1991; 49: 9951013.

6. Jon Soo Kim et al: A case of isodicentric chromosome 15 presented with epilepsy and developmental delay Korean J Pediatr 2012;55(12):487-490

7. Bo Wang.Prenatal diagnosis of small supernumerary marker chromosome 15: A case report. Biomedical Research 2017; 28 (7): 2926-2928.

8. Melo et al. paternal transmission of small supernumerary Marker Chromosome 15 Identified in Prenatal Diagnosis Due to Advanced Maternal Age. Clinical Medicine Insights: Case Reports 2015:8 93-96.
9. Horsthemke B, Wagstaff J. Mechanisms of imprinting of the Prader-Willi/Angelman region. Am J Med Genet A 2008; 146: 2041-2052.

10. Eggermann K, Mau UA, Bujdoso G, Koltai E, Engels $H$. Supernumerary marker chromosomes derived from chromosome 15: analysis of 32 new cases. Clin Genet 200262: 89-93.

11. http://markerchromosomes.ag.vu

12. Su M,Zang $\mathrm{H}$ et al. Molecular characterization of 20 small supernumerary marker chromosome cases using array comparative genomic hybridization and fluorescence in situ hybridizatio.Scientific Reorts[7:10395].

13. Vetro A et al. Unexpected results in the constitution of small supenumerary marker chromosomes.European Journal of Medical Genetics. 2012;55:185-190.

14. Glessner et al.Copy number variation metaanalysis reveals a novel duplication at 9p24 associated with multiple neurodevelopmental disorders. Genome Medicine (2017) 9: 106;p2-11.

15. D Kotzot.Supernumerary marker chromosomes (SMC) and uniparental disomy (UPD): coincidence or consequence. J Med Genet 2002;39:775-778. 